Western Washington University Western CEDAR

2-20-2013

\title{
Comprehensive Analysis of Escape-Cone Losses from Luminescent Waveguides
}

\author{
Stephen R. McDowall \\ Western Washington University, stephen.mcdowall@wwu.edu \\ Tristan Butler \\ Western Washington University \\ Edward Bain \\ Western Washington University \\ Kelsey Scharnhorst \\ Western Washington University \\ David L. Patrick \\ WesternWashington University, david.patrick@wwu.edu
}

Follow this and additional works at: https://cedar.wwu.edu/math_facpubs

Part of the Analysis Commons, and the Analytical Chemistry Commons

\section{Recommended Citation}

McDowall, Stephen R.; Butler, Tristan; Bain, Edward; Scharnhorst, Kelsey; and Patrick, David L., "Comprehensive Analysis of EscapeCone Losses from Luminescent Waveguides" (2013). Mathematics. 32.

https://cedar.wwu.edu/math_facpubs/32 


\title{
Comprehensive analysis of escape-cone losses from luminescent waveguides
}

\author{
Stephen McDowall, ${ }^{1, \star}$ Tristan Butler, ${ }^{2}$ Edward Bain, ${ }^{2}$ Kelsey Scharnhorst, ${ }^{2}$ \\ and David Patrick ${ }^{2}$ \\ 'Department of Mathematics, Western Washington University, 516 High Street, Bellingham, Washington 98225, USA \\ ${ }^{2}$ Department of Chemistry, Western Washington University, 516 High Street, Bellingham, Washington 98225, USA \\ *Corresponding author: stephen.mcdowall@wwu.edu
}

Received 24 October 2012; accepted 21 December 2012;

posted 14 January 2013 (Doc. ID 178603); published 15 February 2013

\begin{abstract}
Luminescent waveguides (LWs) occur in a wide range of applications, from solar concentrators to doped fiber amplifiers. Here we report a comprehensive analysis of escape-cone losses in LWs, which are losses associated with internal rays making an angle less than the critical angle with a waveguide surface. For applications such as luminescent solar concentrators, escape-cone losses often dominate all others. A statistical treatment of escape-cone losses is given accounting for photoselection, photon polarization, and the Fresnel relations, and the model is used to analyze light absorption and propagation in waveguides with isotropic and orientationally aligned luminophores. The results are then compared to experimental measurements performed on a fluorescent dye-doped poly(methyl methacrylate) waveguide. (C) 2013 Optical Society of America
\end{abstract}

OCIS codes: $\quad 230.7370,080.2720,080.5692,030.6600$.

\section{Introduction}

Luminescent waveguides (LWs) are used to capture and guide light in a variety of applications, including luminescent solar concentrators (LSCs) [1-4], scintillation detectors [5], guest-host liquid crystal displays [ $\underline{6}-\underline{8}]$, and doped fiber amplifiers [9]. A typical LW consists of a fluorescent or phosphorescent dye-containing medium having refractive index $n_{1}$ cladded by a second material with refractive index $n_{0}<n_{1}$ (Fig. 1). Externally incident light enters the waveguide and is absorbed by the luminophore, which then emits it in a new direction with a new polarization. The usual intent is to capture as much incident light as possible within the waveguide, causing it to travel by total internal reflection (TIR) to an edge for collection. In practice, however, not all incident light reaches the edge due to several loss mechanisms that combine to reduce the overall

$1559-128 \mathrm{X} / 13 / 061230-10 \$ 15.00 / 0$

(C) 2013 Optical Society of America efficiency: (1) a fraction of externally incident light may be reflected or not absorbed by a luminophore; (2) a fraction of light that is absorbed will not be emitted, determined by the luminophore's quantum yield; and (3) a fraction of emitted light will fail to be trapped by TIR. The latter process is referred to as the escape-cone loss; it occurs for rays intersecting a waveguide surface at angles $\theta_{\mathrm{ph}} \leq \theta_{\mathrm{esc}}$, where $\theta_{\mathrm{ph}}$ is the polar angle of the ray with respect to the surface normal and the critical angle is given by Snell's Law: $\theta_{\text {esc }}=\sin ^{-1}\left(n_{0} / n_{1}\right)$. In a large LW, escape-cone losses are often the single most important mechanism limiting efficiency. Compounding its effect, light traveling within the waveguide may encounter other luminophores, to be reabsorbed and reemitted multiple times. With each such occurrence there is a repeated chance of escape-cone loss.

In the case of LSCs-the LW application we focus on here-escape-cone losses play a particularly important role. LSCs are planar LWs used to collect and concentrate sunlight for conversion into electricity by photovoltaic cells attached around the waveguide 
edges. By concentrating sunlight with a relatively inexpensive sheet of luminescent plastic or glass, the photovoltaic cell area-and hence the cost-is reduced. Further advantages of LSCs include their ability to concentrate both diffuse and specular light without the need for solar tracking, the potential for achieving very large concentration factors, and delivery to the photovoltaic cells of wavelength-to-bandgap matched photons. First proposed over 30 years ago [1-ㅁ], losses from mechanisms (1) and (2) above can in principle be controlled through a combination of antireflection coatings and the use of strongly absorbing, highly luminescent dyes. Yet the efficiency of LSCs still remains too low for practical applications, largely as a result of escape-cone losses [10-13].

Despite its importance for LSCs and related technologies, it appears that a complete treatment of escape-cone losses in LWs has yet to be given. Instead, the usual approach, first described by Shurcliff and Jones [14], and used by almost all workers since, is to assume the per-emission escape-cone loss rate simply equals the proportion of the solid angle defined by the critical cone, $\mathcal{C}$, out of the full solid angle of the sphere, $4 \pi$. That is,

$$
P(\mathrm{esc})=1-\sqrt{1-\left(n_{0} / n_{1}\right)^{2}} .
$$

For a poly(methyl methacrylate) (PMMA) waveguide cladded by air $\left(n_{1}=1.49, n_{0}=1\right)$, Eq. (1) gives $P($ esc $) \approx 25.9 \%$. This result, however, is only correct in the case of isotropic emission, as would occur, for example, if the LW contained randomly oriented luminophores undergoing rapid rotational diffusion on the time scale of the excited-state lifetime. In most instances-particularly in solid media-such rotation is actually relatively slow, and consequently the probability distribution function for the direction and polarization of an emitted photon is not independent of the direction and polarization of the exciting photon. Stated differently, Eq. (1) neglects the important role of photoselection. Additional deviations also result from consideration of the Fresnel relations, although in LSCs and related LWs, their effect is quite small. Finally, there can also be cases in which the luminophores are not randomly oriented. For example, in certain types of LSCs, as well as in related systems such as dichroic liquid crystal displays and liquid crystal lasers, the dye molecules are imbedded in a macroscopically oriented matrix such as a liquid crystal, reactive mesogen, or stretched polymer. This aligns the luminophores along a preferred axis, breaking the rotational symmetry presumed in Eq. (1).

In the following we provide the first treatment, to our knowledge, of escape-cone losses in LWs fully accounting for these phenomena. We consider both the effects of photoselection and luminophore alignment, finding each to cause significant deviations from the predictions of Eq. (1). The results are then compared to experimental measurements of escapecone losses performed on an LW consisting of a
PMMA slab doped with low concentrations of a fluorescent red dye. We find agreement between the theory and experiment at the $95 \%$ confidence level.

Before proceeding to a discussion of the full theoretical treatment and experimental measurements, we first illustrate the nature of some of the results. Consider an LW containing randomly oriented dye molecules having coincident absorption and emission dipoles. Assume also that the waveguide has a geometry like that shown in Fig. 1 and is illuminated perpendicular to the plane by unpolarized light, and that the host medium has an index of refraction $n_{1}=1.49$ (e.g., PMMA) and is cladded by air. Based on the theory developed below, we calculate the probability of a photon being emitted from a molecule that has absorbed such incident light into the escape cone; we shall call this the first-emission escapecone loss. We then calculate the probability distribution for the direction and polarization of the light not lost out the escape cone. Conditional on the fact that the surviving light is distributed thus, we compute the probability that such light is absorbed and emitted out the escape cone again, and so on. For such a case we find the following.

The first-emission escape-cone loss is $29.2 \%$. If we assume that all emitted light, including rays traveling within the escape cone, has a chance of being absorbed and then emitted (i.e., that the luminophore concentration is very high), then the secondemission escape cone loss is $26.7 \%$. Maintaining this assumption that all emitted photons can be reabsorbed, the third- and fourth-emission escape-cone losses are, respectively, $25.6 \%$ and $25.4 \%$. Escape-cone losses are leveling out at approximately the same as one would obtain using Eq. (1), namely $25.9 \%$.

Perhaps a more pertinent computation, however, is to assume that light traveling in a direction that will cause it to be lost out the escape cone is indeed lost, as would be the case if the concentration of the luminescent species were not too high. Again for randomly oriented luminophores, we find the following.

The first-emission escape-cone loss is $29.2 \%$. If we now compute the probability of photons escaping from a second emission event, conditional on the fact that the absorbed photon had a direction outside the

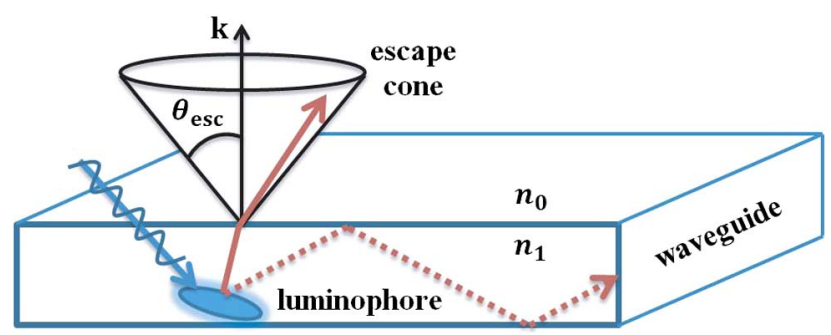

Fig. 1. (Color online) Typical geometry of a LW: incoming light is absorbed by a lumninophore and when emitted may be emitted in a direction resulting in TIR (dashed red ray) or in a direction resulting in loss out the escape cone (solid red ray). When used as an LSC, photovoltaic cells are placed around the waveguide edge to convert captured light into electricity. 
escape cone (from the first emission event), then the second-emission escape-cone loss is $26.1 \%$. Continuing under this assumption, we obtain escape-cone losses of $24.1 \%, 24.0 \%$, and $24.0 \%$ for the thirdthrough fifth-emission events.

We also illustrate some results for LWs having nonrandom luminophore orientation. Recent experimental [15-19] and theoretical [20] findings suggest that LSCs incorporating orientationally aligned dyes may improve performance by reducing escape-cone losses, and our detailed results below support this conclusion. Oriented LWs are important in other applications as well, such as liquid crystal lasers [21]. To demonstrate the effect of orientation on escapecone losses, consider an LW in which luminophores are uniaxially distributed about a normal vector $\mathbf{k}$ to the planar interface of a slab waveguide (refer to Fig. 1). If the angle $\beta$ between the dye molecule's absorption and emission transition moments $\boldsymbol{\mu}_{\mathrm{abs}}$ and $\boldsymbol{\mu}_{\mathrm{em}}$ is small, then orienting the luminophores in this way decreases the escape-cone loss rate because more light tends to be emitted in the plane of the LSC (see below for details). (The angle $\beta$ is related to a luminophore's fundamental anisotropy, $r_{0}$ through $r_{0}=\left(3 \cos ^{2} \beta-1\right) / 5$. Most organic dyes with high fluorescent quantum yields have $\beta \lesssim 20^{\circ}$ [22].) The degree of orientational order is defined in terms of an order parameter, taken as the ensemble average of the second Legendre polynomial of the cosine of the angle between $\boldsymbol{\mu}_{\mathrm{abs}}$ and $\mathbf{k}: P 2=\left\langle 3 / 2\left(\boldsymbol{\mu}_{\mathrm{abs}} \cdot \mathbf{k}\right)^{2}-\right.$

Table 1. Proportion of Light Lost Out of the Escape Cone at Successive Absorption/Emission Events for Various Degrees of Luminophore Alignment ${ }^{a}$

\begin{tabular}{rrrccc}
\hline P2 & 1st (\%) & 2nd (\%) & 3rd (\%) & 4th (\%) & 5th (\%) \\
\hline 0.0 & 29.2 & 26.1 & 24.1 & 24.0 & 24.0 \\
0.5 & 23.4 & 17.3 & 15.8 & 15.6 & 15.6 \\
0.6 & 21.5 & 15.5 & 14.5 & 14.5 & 14.5 \\
0.7 & 19.1 & 13.8 & 13.3 & 13.3 & 13.3 \\
0.8 & 16.0 & 12.2 & 12.1 & 12.1 & 12.1 \\
1.0 & 9.2 & 9.2 & 9.2 & 9.2 & 9.2 \\
\hline
\end{tabular}

${ }^{a}$ Computed using the model described in the text, based on a PMMA waveguide cladded by air, neglecting self-absorption, and accounting for Fresnel relations.

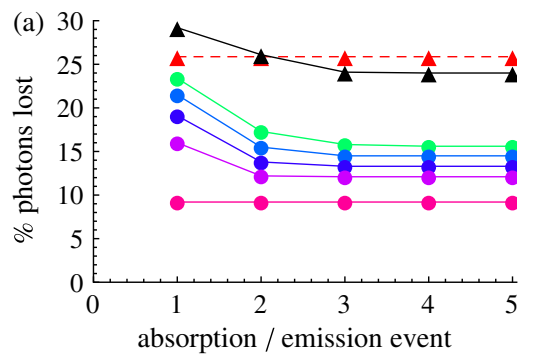

$1 / 2\rangle$, where $\langle\cdot\rangle$ denotes an average over all luminophores. For the cases we will consider, the function $P 2$ varies between 1 and 0 for perfectly ordered and perfectly random alignment, respectively. The value of $P 2$ can also be negative, as would be the case if individual luminophores tended to orient themselves orthogonal to $\mathbf{k}$. The analogous computations to the second case above are then performed for varying $P 2$ statistics, giving, for each successive reabsorption/ reemission event, the results in Table $\underline{1}$.

The above-described results are summarized in Fig. 2(a), which shows the proportion of surviving light lost to the escape cone at the $j$ th event for isotropic dyes and for dyes with varying degrees of alignment. The solid triangles are for isotropic dyes; the solid circles are for oriented dyes, with $P 2$ varying from 0.5 (top) through 1.0 (bottom). Also shown, as the top dashed line, is the simplistic assumption embodied in Eq. (1) that $25.9 \%$ of light is lost at every event. Figure 2(b) shows the corresponding overall proportion of light remaining after $j$ absorption/emission events.

We draw attention to several features of these results. First, the escape cone loss probability is always highest for the first emission event, decreasing thereafter before leveling out at large $j$. This behavior results from including the effects of photoselection and the assumption that $\beta$ is small; $P($ esc $)$ is largest for the first emission because normally incident light preferentially excites luminophores having $\boldsymbol{\mu}_{\mathrm{abs}}$ (and hence $\boldsymbol{\mu}_{\mathrm{em}}$ ) closer to the LW plane. Thereafter, surviving rays (i.e., those not lost out the escape cone) are more likely to be traveling in directions making larger angles to $\mathbf{k}$, and hence to photoselect dye molecules oriented with $\boldsymbol{\mu}_{\mathrm{abs}}$ more parallel to $\mathbf{k}$. As this process repeats, a steady-state escape probability is eventually approached.

Further insight into this process can be gained by considering the orientational statistics of the subpopulations of luminescent species participating in successive generations of absorption/emission events. This we quantify in Fig. 3 , which shows $P 2^{*}(j)$ for an LW with randomly oriented luminophores (the asterisk denotes that the computation is performed only on those dye molecules absorbing and emitting a

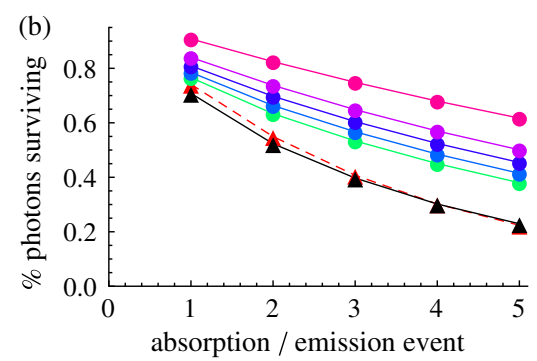

Fig. 2. (Color online) Successive absorption/emission events for LWs containing isotropic (triangles) and oriented (circles) luminophores. The prediction of Eq. (1) is shown by the dashed lines, based on a PMMA waveguide cladded by air, neglecting self-absorption and accounting for Fresnel relations. Triangles plus dashed line: simplistic assumption given by Eq. (1); triangles plus solid line: present model assuming isotropically oriented luminophores; circles plus solid lines: present model assuming oriented luminophores with $P 2=0.5,0.6, \ldots, 1.0$. (a) Proportion of absorbed light lost out the escape cone at successive absorption/emission events. (b) Proportion of light remaining within the LW at successive absorption/emission events. 


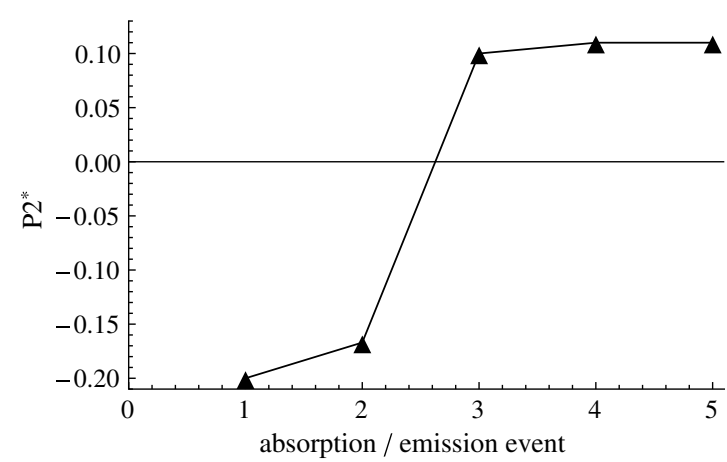

Fig. 3. Order parameters of photoselected dye molecules, by generation, for an LW with randomly oriented luminophores and $n_{1}=1.49, n_{0}=1, \beta=0$.

photon in the respective generation). In the first generation, external normally incident light photoselects dyes oriented in the LW plane, and hence $P 2^{*}(j=1)<0$. The orientational statistics of the subpopulation of participating molecules evolves with successive generations, approaching a steadystate value somewhat above $P 2^{*}=0$.

Returning to Figs. 2(a) and 2(b), also noteworthy is the significant reduction in escape-cone losses seen to be achievable by orienting the luminophores. Taking $P 2=0.8$ as an example, $P($ esc $)$ is reduced by half relative to an LW with randomly oriented dye molecules. Note though that escape-cone losses do not approach zero even for a perfectly ordered system $(P 2=1)$. This is because the probability distribution function for $\alpha$, the angle between the photon emission directions and $\boldsymbol{\mu}_{\mathrm{em}}$, is equal to $3 /(8 \pi) \sin ^{2} \alpha \mathrm{d} A$ (where $\mathrm{d} A$ is the area measure on the sphere). Hence a fraction of light $P(\mathrm{esc}, P 2=1)=3 /(4 \pi) \int_{0}^{2 \pi} \int_{0}^{\theta_{\text {esc }}}$ $\sin ^{2} \theta \sin \theta \mathrm{d} \theta \mathrm{d} \varphi \approx 0.092$, for $n_{1}=1.49$ and $\beta=0$, lies within the escape cone even in a system of perfectly ordered dye molecules.

\section{Analytic Derivations}

In this article, we are interested in the proportion of photons emitted from an isotropic dye that will lie in the escape cone $\mathcal{C}$ relative to the normal vector $\mathbf{k}$ to the planar interface with air. We are interested in the dependence on the velocity vector $\mathbf{v}$ of the absorbed photon (specifically on the angle between $\mathbf{v}$ and $\mathbf{k}$ ) and on the polarization $\mathbf{p}$ of the absorbed photon. For unpolarized light, we will compute the integral over all polarizations $\mathbf{p}$ (perpendicular to $\mathbf{v}$ ). The absorbing dye is assumed to have an interdipole angle $\beta$, defined above as the angle between the absorption and emission moments of the absorbing luminophore.

Let $\mathbf{v}_{\mathrm{abs}}$ and $\mathbf{v}_{\mathrm{em}}$ be the velocity vectors of the incoming (absorbed) and outgoing (emitted) photon. Because the probability that $\mathbf{v}_{\mathrm{em}}$ lies in $\mathcal{C}, P\left(\mathbf{v}_{\mathrm{em}} \in \mathcal{C}\right)$, is rotationally symmetric about $\mathbf{k}$ we may assume, without loss of generality, that

$$
\mathbf{v}_{\mathrm{abs}}=\mathbf{v}_{\mathrm{abs}}\left(\theta_{\mathrm{ph}}\right)=\left(\sin \theta_{\mathrm{ph}}, 0, \cos \theta_{\mathrm{ph}}\right) .
$$

Here, $\theta_{\mathrm{ph}}$ is the polar angle of the absorbed photon. Recall that the escape cone $\mathcal{C}$ is the cone of vectors making an angle of less than $\theta_{\text {esc }}$ with $\mathbf{k}$, where $\sin \theta_{\text {esc }}=n_{0} / n_{1}$. We are principally interested in this being air, $n_{0}=1$. An emitted photon with $\mathbf{v}_{\text {em }} \in \mathcal{C}$ can refract out of the substrate, that is, it will escape. The polarization $\mathbf{p}$ of the incoming photon is perpendicular to $\mathbf{v}_{\mathrm{abs}}$ and so can be parameterized by

$$
\begin{aligned}
\mathbf{p}_{\mathrm{abs}} & =\mathbf{p}_{\mathrm{abs}}\left(\theta_{\mathrm{ph}}, \alpha_{p}\right) \\
& =\cos \alpha_{p}\left(\cos \theta_{\mathrm{ph}}, 0,-\sin \theta_{\mathrm{ph}}\right)+\sin \alpha_{p}(0,1,0) .
\end{aligned}
$$

We parameterize the set of emission dipoles $\boldsymbol{\mu}_{\mathrm{em}}$ by spherical coordinates,

$$
\begin{aligned}
\boldsymbol{\mu}_{\mathrm{em}} & =\boldsymbol{\mu}_{\mathrm{em}}\left(\theta_{\mu_{e}}, \varphi_{\mu_{e}}\right) \\
& =\left(\sin \theta_{\mu_{e}} \cos \varphi_{\mu_{e}}, \sin \theta_{\mu_{e}} \sin \varphi_{\mu_{e}}, \cos \theta_{\mu_{e}}\right) .
\end{aligned}
$$

We seek to determine $P\left(\operatorname{esc} \mid \mathbf{p}_{\mathrm{abs}}, \mathbf{v}_{\mathrm{abs}}\right)$, the probability that an emitted photon escapes the LW, given that it began with incoming direction and polarization $\mathbf{v}_{\mathrm{abs}}$ and $\mathbf{p}_{\mathrm{abs}}$. If we denote by $P\left(\operatorname{esc} \mid \boldsymbol{\mu}_{\mathrm{em}}\right)$ the probability that a photon escapes conditional on its being emitted by a luminophore with emission dipole $\boldsymbol{\mu}_{\mathrm{em}}$, and by $P\left(\mu_{\mathrm{em}} \mid \mathbf{p}_{\mathrm{abs}}, \mathbf{v}_{\mathrm{abs}}\right)$ the probability that the photon was emitted from a dipole $\boldsymbol{\mu}_{\mathrm{em}}$ conditional on the incoming photon's velocity and polarization vectors, then

$$
\begin{aligned}
P\left(\operatorname{esc} \mid \mathbf{p}_{\mathrm{abs}}, \mathbf{v}_{\mathrm{abs}}\right)= & \int_{\mathbb{S}^{2}} P\left(\mathrm{esc} \mid \boldsymbol{\mu}_{\mathrm{em}}\right) \\
& \times P\left(\boldsymbol{\mu}_{\mathrm{em}} \mid \mathbf{p}_{\mathrm{abs}}, \mathbf{v}_{\mathrm{abs}}\right) \mathrm{d} A\left(\boldsymbol{\mu}_{\mathrm{em}}\right) .
\end{aligned}
$$

The measure $\mathrm{d} A\left(\mu_{\mathrm{em}}\right)$ is the area measure on the sphere; in our spherical coordinates it is $\mathrm{d} A\left(\mu_{\mathrm{em}}\right)=$ $\sin \theta_{\mu_{e}} \mathrm{~d} \theta_{\mu_{e}} \mathrm{~d} \varphi_{\mu_{e}}$. In the following, we derive expressions for the two terms in the integrand of Eq. (ㅁ). The first represents the probability of a photon escaping, conditional on the fact that it has been emitted from a specific emission dipole. This probability depends on the orientation of the dipole, on the emission profile (assumed to be dipolar here; see below), and on the Fresnel relations. The second term is the probability that emission has occurred from a given dipole, given the velocity and polarization vectors of the incident photon. Together, the two terms fully account for photoselection and the luminescent anisotropy through the inclusion of $\beta$.

We begin with the first term in the integrand of Eq. (ㅁ), which is given by

$$
P\left(\operatorname{esc} \mid \boldsymbol{\mu}_{\mathrm{em}}\right)=\int_{\mathbb{S}^{2}} P\left(\mathrm{esc} \mid \mathbf{v}_{\mathrm{em}}\right) P\left(\mathbf{v}_{\mathrm{em}} \mid \boldsymbol{\mu}_{\mathrm{em}}\right) \mathrm{d} A\left(\mathbf{v}_{\mathrm{em}}\right),
$$

where $P\left(\mathrm{esc} \mid \mathbf{v}_{\mathrm{em}}\right)$ is the probability that an emitted photon with velocity $\mathbf{v}_{\mathrm{em}}$ escapes and is determined by the Fresnel relations if $\mathbf{v}_{\mathrm{em}} \in \mathcal{C}$ and is zero otherwise, and where $P\left(\mathbf{v}_{\mathrm{em}} \mid \boldsymbol{\mu}_{\mathrm{em}}\right)$ is the probability of a photon emitted from $\boldsymbol{\mu}_{\mathrm{em}}$ having direction $\mathbf{v}_{\mathrm{em}}$. Treating the luminophores as dipolar radiators (far-field 
approximation) [23], the latter is proportional to the square of the sine of the angle $\sigma$ between $\mathbf{v}_{\mathrm{em}}$ and $\boldsymbol{\mu}_{\mathrm{em}}$ but is uniform in the circle of vectors on the sphere making this angle. To compute Eq. (ㅁ) we parameterize the vectors $\mathbf{v}_{\mathrm{em}}$ by the angle $\sigma$ and a parameterization in $\rho$ of the circle determined by $\sigma$ : for $\boldsymbol{\mu}_{\mathrm{em}} \neq \mathbf{k}$,

$$
\begin{aligned}
\mathbf{v}_{\mathrm{em}}\left(\sigma, \rho ; \boldsymbol{\mu}_{\mathrm{em}}\right)= & \sin \sigma\left[\cos \rho\left(\frac{\boldsymbol{\mu}_{\mathrm{em}} \times \mathbf{k}}{\left\|\boldsymbol{\mu}_{\mathrm{em}} \times \mathbf{k}\right\|}\right) \times \boldsymbol{\mu}_{\mathrm{em}}\right. \\
& \left.+\sin \rho \frac{\boldsymbol{\mu}_{\mathrm{em}} \times \mathbf{k}}{\left\|\boldsymbol{\mu}_{\mathrm{em}} \times \mathbf{k}\right\|}\right]+\cos \sigma \boldsymbol{\mu}_{\mathrm{em}} .
\end{aligned}
$$

If $\boldsymbol{\mu}_{\mathrm{em}}=\mathbf{k}$, then we may take $\mathbf{v}_{\mathrm{em}}(\sigma, \rho ; \mathbf{k})=$ $(\sin \sigma \cos \rho, \sin \sigma \sin \rho, \cos \sigma)$. With this parameterization,

$$
\begin{aligned}
P\left(\mathbf{v}_{\mathrm{em}} \mid \boldsymbol{\mu}_{\mathrm{em}}\right) \mathrm{d} A\left(\mathbf{v}_{\mathrm{em}}\right) & =\frac{\sin ^{2} \sigma}{\int_{\mathbb{S}^{2}}\left(1-\left(\mathbf{v} \cdot \boldsymbol{\mu}_{\mathrm{em}}\right)^{2}\right) \mathrm{d} A(\mathbf{v})} \sin \sigma \mathrm{d} \sigma \mathrm{d} \rho \\
& =\frac{3}{8 \pi} \sin ^{3} \sigma \mathrm{d} \sigma \mathrm{d} \rho .
\end{aligned}
$$

The probability in Eq. (ㅁ) can now be expressed as

$$
\begin{aligned}
P\left(\operatorname{esc} \mid \boldsymbol{\mu}_{\mathrm{em}}\right)= & \frac{3}{8 \pi} \int_{0}^{\pi}\left(\int_{-\pi}^{\pi} P\left(\operatorname{esc} \mid \mathbf{v}_{\mathrm{em}}\left(\sigma, \rho ; \boldsymbol{\mu}_{\mathrm{em}}\right)\right) \mathrm{d} \rho\right) \\
& \times \sin ^{3} \sigma \mathrm{d} \sigma .
\end{aligned}
$$

We proceed to compute $P\left(\operatorname{esc} \mid \boldsymbol{\mu}_{\mathrm{em}}\left(\sigma, \rho ; \boldsymbol{\mu}_{\mathrm{em}}\right)\right)$. In Eq. (5) we can express the integral as twice that over $0 \leq \theta_{\mathrm{em}} \leq \pi / 2$ and so need only consider such $\theta_{\mathrm{em}}$. We can also compute the probability of escape out the half-cone $\mathcal{C}_{+}$about $\mathbf{k}$ and take twice this quantity because the process is symmetric about the plane perpendicular to $\mathbf{k}$. We shall determine an expression for the function $R_{+}\left(\theta_{\mathrm{em}}, \sigma\right)$, which is such that $\mathbf{v}_{\mathrm{em}}\left(\sigma, \rho ; \boldsymbol{\mu}_{\mathrm{em}}\right) \in \mathcal{C}_{+}$for $|\rho|<R_{+}\left(\theta_{\mathrm{em}}, \sigma\right)$; we integrate over this range $\left(-\rho<R_{+}<\rho\right)$ the probability of escaping, as determined by the Fresnel relations.

The situation differs depending on whether $\theta_{\mathrm{em}}$ is greater than, or less than, $\theta_{\text {esc }}$. Suppose first that $\theta_{\mathrm{em}} \geq \theta_{\mathrm{esc}}$. When $\sigma \notin\left[\theta_{\mathrm{em}}-\theta_{\mathrm{esc}}, \theta_{\mathrm{em}}+\theta_{\mathrm{esc}}\right]$, $R_{+}\left(\theta_{\mathrm{em}}, \sigma\right)=0$. Otherwise, Figs. 4(a) and 4(b) below show the two typical configurations with respect to

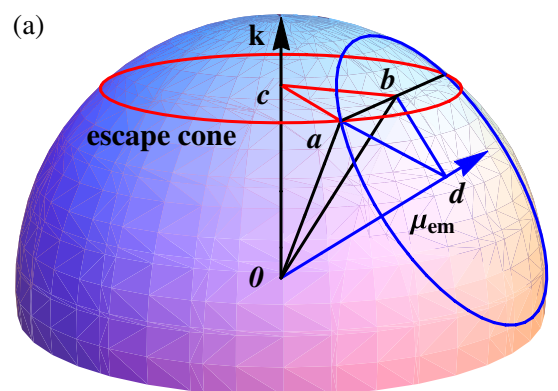

$\mathcal{C}_{+}$. Referring to these figures, we need to determine the angle $R_{+}\left(\theta_{\mathrm{em}}, \sigma\right)=\angle a \mathrm{~d} b$ :

Because $|a \mathrm{~d}|=\sin \sigma$, it suffices to determine $|b \mathrm{~d}|$. We have $|0 \mathrm{~d}|=\cos \sigma,|0 c|=\cos \theta_{\text {esc }}$, and the angle $\angle c 0 \mathrm{~d}=\theta_{\mathrm{em}}$. Thus we may consider the quadrilateral $0 c b \mathrm{~d}$, which takes one of the two forms shown in Figs. 5(a) and 5(b).

It is straightforward to derive $|b \mathrm{~d}|=\left(\cos \theta_{\mathrm{esc}^{-}}\right.$ $\left.\cos \sigma \cos \theta_{\mathrm{em}}\right) / \sin \theta_{\mathrm{em}}$. Thus, from the right triangle $a \mathrm{~d} b$,

$$
R_{+}\left(\theta_{\mathrm{em}}, \sigma\right)=\arccos \left(\frac{\cos \theta_{\mathrm{esc}}-\cos \sigma \cos \theta_{\mathrm{em}}}{\sin \theta_{\mathrm{em}} \sin \sigma}\right) .
$$

When $\theta_{\mathrm{em}}<\theta_{\mathrm{esc}}$, once again $R_{+}\left(\theta_{\mathrm{em}}, \sigma\right)=0$ for $\sigma>$ $\theta_{\mathrm{em}}+\theta_{\mathrm{esc}}$, and Eq. (10) still holds when $\theta_{\mathrm{esc}}-\theta_{\mathrm{em}}<$ $\sigma<\theta_{\text {esc }}+\theta_{\text {em }}$ [see Fig. 6(a)]; for $0<\sigma<\theta_{\text {esc }}-\theta_{\text {em }}$ we simply define $R_{+}\left(\theta_{\mathrm{em}}, \sigma\right)=\pi$ [see Fig. $\underline{6(\mathrm{~b})}$ ] .

Having defined $R_{+}$, we now incorporate the Fresnel relations. If an emitted photon has $\mathbf{v}_{\mathrm{em}}=$ $\mathbf{v}_{\mathrm{em}}\left(\sigma, \rho ; \boldsymbol{\mu}_{\mathrm{em}}\right) \in \mathcal{C}$, then the photon's polarization is given by

$$
\mathbf{p}_{\mathrm{em}}\left(\sigma, \rho ; \boldsymbol{\mu}_{\mathrm{em}}\right)=\frac{\mathbf{v}_{\mathrm{em}} \times\left(\mathbf{v}_{\mathrm{em}} \times \boldsymbol{\mu}_{\mathrm{em}}\right)}{\left\|\mathbf{v}_{\mathrm{em}} \times\left(\mathbf{v}_{\mathrm{em}} \times \boldsymbol{\mu}_{\mathrm{em}}\right)\right\|} .
$$

To compute the probability that such a photon is reflected at the boundary of the waveguide, we decompose $\mathbf{p}$ into its $s$-polarized and $p$-polarized components, define $\mathbf{s}=\left(\mathbf{k} \times \mathbf{v}_{\mathrm{em}}\right) /\left\|\mathbf{k} \times \mathbf{v}_{\mathrm{em}}\right\|$, and write

$$
\mathbf{p}_{\mathrm{em}}=\left(\mathbf{p}_{\mathrm{em}} \cdot \mathbf{s}\right) \mathbf{s}+\left(\mathbf{p}_{\mathrm{em}} \cdot\left(\mathbf{v}_{\mathrm{em}} \times \mathbf{s}\right)\right)\left(\mathbf{v}_{\mathrm{em}} \times \mathbf{s}\right) .
$$

The probability of reflection is then

$$
R_{s}\left(\mathbf{v}_{\mathrm{em}}\right)\left(\mathbf{p}_{\mathrm{em}} \cdot \mathbf{s}\right)^{2}+R_{p}\left(\mathbf{v}_{\mathrm{em}}\right)\left(\mathbf{p}_{\mathrm{em}} \cdot\left(\mathbf{v}_{\mathrm{em}} \times \mathbf{s}\right)\right)^{2},
$$

where

$$
\begin{gathered}
R_{s}(\mathbf{v})=\left(\frac{h(\mathbf{v})-n_{1} / n_{0}}{h(\mathbf{v})+n_{1} / n_{0}}\right)^{2}, \\
R_{p}(\mathbf{v})=\left(\frac{h(\mathbf{v})-n_{0} / n_{1}}{h(\mathbf{v})+n_{0} / n_{1}}\right)^{2}, \\
h(\mathbf{v})=\frac{1}{\mathbf{v} \cdot \mathbf{k}} \sqrt{1-\left(n_{1} / n_{0}\right)^{2}\left(1-(\mathbf{v} \cdot \mathbf{k})^{2}\right)} .
\end{gathered}
$$

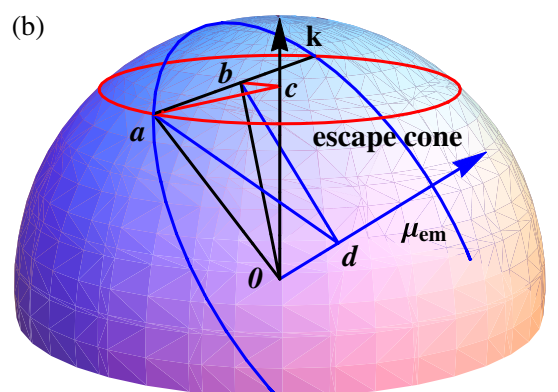

Fig. 4. (Color online) Description of the computational geometry when $\theta_{\mathrm{em}} \geq \theta_{\mathrm{esc}}$. (a) Case $\theta_{\mathrm{em}}-\theta_{\mathrm{esc}}<\sigma<\theta_{\mathrm{em}}$. (b) Case $\theta_{\mathrm{em}}<\sigma<\theta_{\mathrm{em}}<\theta_{\mathrm{esc}}$. 
(a)

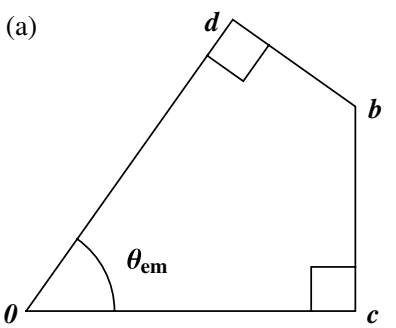

(b)

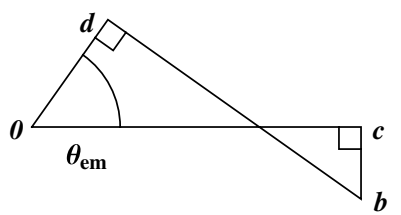

Fig. 5. Geometry involved in the computation of $|b \mathrm{~d}|$. (a) Case $\sigma<\theta_{\text {esc }}$. (b) Case $\sigma>\theta_{\text {esc }}$.

Recalling that we compute only for $\mathbf{v}_{\mathrm{em}} \in \mathcal{C}_{+}$, Eq. (9) becomes, with $\mathbf{v}_{\mathrm{em}}=\mathbf{v}_{\mathrm{em}}\left(\sigma, \rho ; \boldsymbol{\mu}_{\mathrm{em}}\right)$ and $\mathbf{p}_{\mathrm{em}}=$ $\mathbf{p}_{\mathrm{em}}\left(\bar{\sigma} \rho ; \boldsymbol{\mu}_{\mathrm{em}}\right)$,

$$
\begin{aligned}
P\left(\operatorname{esc} \mid \boldsymbol{\mu}_{\mathrm{em}}\right)= & \frac{3}{4 \pi} \int_{0}^{\pi} \int_{-R_{+}\left(\theta_{\mathrm{em}}, \sigma\right)}^{R_{+}\left(\theta_{\mathrm{em}}, \sigma\right)}\left[1-R_{s}\left(\mathbf{v}_{\mathrm{em}}\right)\left(\mathbf{p}_{\mathrm{em}} \cdot \mathbf{s}\right)^{2}\right. \\
& \left.-R_{p}\left(\mathbf{v}_{\mathrm{em}}\right)\left(\mathbf{p}_{\mathrm{em}} \cdot\left(\mathbf{v}_{\mathrm{em}} \times \mathbf{s}\right)\right)^{2}\right] \mathrm{d} \rho \sin ^{3} \sigma \mathrm{d} \sigma .
\end{aligned}
$$

The probability in Eq. (16) is independent of $\varphi_{\mathrm{em}}$ and so can be computed (numerically) as a function of $\theta_{\mathrm{em}}$. This is shown as the solid curve in Fig. 7 (with $n_{0}=1$ and $n_{1}=1.49$ ). The dashed curve shows the same probability when the Fresnel relations are not taken into account; in this case, any photon within the escape cone necessarily escapes. This probability can be computed analytically as follows: the integrand of Eq. (16) is replaced by the constant 1 and $P\left(\operatorname{esc} \mid \mu_{\mathrm{em}}\right)$ is recognized as $3 / 4 \pi$ times the area integral over the escape cone, weighted by $\sin ^{2} \sigma, \sigma$ being the angle between $\mathbf{v}_{\mathrm{em}}$ and $\boldsymbol{\mu}_{\mathrm{em}}$. Given this realization, we can reparameterize the integral by fixing $\boldsymbol{\mu}_{\mathrm{em}}=\left(\cos \theta_{\mathrm{em}}, 0\right.$, sin $\left.\theta_{\mathrm{em}}\right)$ and parameterizing $\mathbf{v}_{\mathrm{em}}$ in standard polar coordinates $(\theta, \varphi)$. Then $\sin ^{2} \sigma \mathrm{d} A$ becomes $\left(1-\left(\mathbf{v}_{\mathrm{em}} \cdot \boldsymbol{\mu}_{\mathrm{em}}\right)^{2}\right) \sin \theta \mathrm{d} \varphi \mathrm{d} \theta$. Computing, we find

$$
\begin{aligned}
P\left(\mathrm{esc} \mid \boldsymbol{\mu}_{\mathrm{em}}\right)= & \left(1-\frac{33}{32} \cos \theta_{\mathrm{esc}}+\frac{1}{32} \cos \left(3 \theta_{\mathrm{esc}}\right)\right) \\
& -\frac{3}{32}\left(\cos \theta_{\mathrm{esc}}-\cos \left(3 \theta_{\mathrm{esc}}\right)\right) \cos \left(2 \theta_{\mathrm{em}}\right) \\
= & 0.217-0.125 \cos \left(2 \theta_{\mathrm{em}}\right) \\
\text { when } n_{0}= & 1 \text { and } n_{1}=1.49 .
\end{aligned}
$$

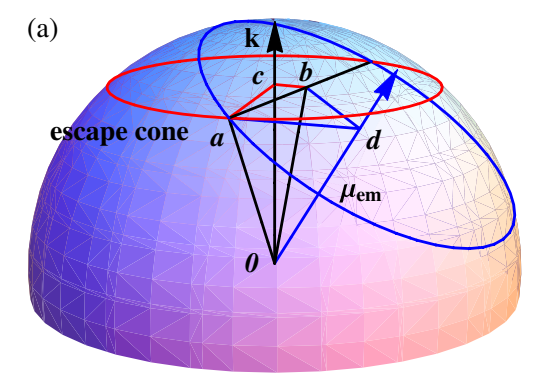

Returning to Eq. (5), we now turn to finding $P\left(\boldsymbol{\mu}_{\mathrm{em}} \mid \mathbf{p}_{\mathrm{abs}}, \mathbf{v}_{\mathrm{abs}}\right)$. In order to be emitted by a dipole $\boldsymbol{\mu}_{\mathrm{em}}$, the photon must be absorbed by a dipole $\boldsymbol{\mu}_{\mathrm{abs}}$ that makes an angle $\beta$ with $\boldsymbol{\mu}_{\mathrm{em}}$. If $\boldsymbol{\mu}_{\mathrm{abs}}(\tau)$ is a parameterization of this circle of vectors, then

$P\left(\boldsymbol{\mu}_{\mathrm{em}} \mid \mathbf{p}_{\mathrm{abs}}, \mathbf{v}_{\mathrm{abs}}\right)=\frac{q}{2 \pi} \int_{\mathbb{S}} P\left(\boldsymbol{\mu}_{\mathrm{abs}}(\tau) \mid \mathbf{p}_{\mathrm{abs}}, \mathbf{v}_{\mathrm{abs}}\right) \mathrm{d} s(\tau)$.

Here, $q$ is the quantum yield of the dye ( $q \leq 1)$, which is the probability of emission, and $P\left(\boldsymbol{\mu}_{\mathrm{abs}}(\tau) \mid \mathbf{p}_{\mathrm{abs}}, \mathbf{v}_{\mathrm{abs}}\right)$ is the probability of absorption by $\mu_{\text {abs }}(\tau)$, conditional on $\mathbf{p}_{\mathrm{abs}}, \mathbf{v}_{\mathrm{abs}}$. By Bayes's theorem,

$$
\begin{aligned}
& P\left(\boldsymbol{\mu}_{\mathrm{abs}}(\tau) \mid \mathbf{p}_{\mathrm{abs}}, \mathbf{v}_{\mathrm{abs}}\right) \\
& \quad=\frac{P\left(\text { absorption } \mid \boldsymbol{\mu}_{\mathrm{abs}}(\tau), \mathbf{p}_{\mathrm{abs}}, \mathbf{v}_{\mathrm{abs}}\right)}{\int_{\mathbb{S}^{2}} P\left(\text { absorption } \mid \boldsymbol{\mu}, \mathbf{p}_{\mathrm{abs}}, \mathbf{v}_{\mathrm{abs}}\right) \mathrm{d} A(\boldsymbol{\mu})} .
\end{aligned}
$$

Now $P\left(\right.$ absorption $\left.\mid \boldsymbol{\mu}_{\text {abs }}(\tau), \mathbf{p}_{\text {abs }}, \mathbf{v}_{\text {abs }}\right) \propto\left(\boldsymbol{\mu}_{\text {abs }}(\tau) \cdot \mathbf{p}_{\text {abs }}\right)^{2}$, so

$$
\begin{aligned}
P\left(\boldsymbol{\mu}_{\mathrm{abs}}(\tau) \mid \mathbf{p}_{\mathrm{abs}}, \mathbf{v}_{\mathrm{abs}}\right) & =\frac{\left(\boldsymbol{\mu}_{\mathrm{abs}}(\tau) \cdot \mathbf{p}_{\mathrm{abs}}\right)^{2}}{\int_{\mathbb{S}^{2}}\left(\boldsymbol{\mu} \cdot \mathbf{p}_{\mathrm{abs}}\right)^{2} \mathrm{~d} A(\boldsymbol{\mu})} \\
& =\frac{3}{4 \pi}\left(\boldsymbol{\mu}_{\mathrm{abs}}(\tau) \cdot \mathbf{p}_{\mathrm{abs}}\right)^{2} .
\end{aligned}
$$

Thus Eq. (18) becomes

$$
P\left(\boldsymbol{\mu}_{\mathrm{em}} \mid \mathbf{p}_{\mathrm{abs}}, \mathbf{v}_{\mathrm{abs}}\right)=\frac{3 q}{8 \pi^{2}} \int_{\mathbb{S}}\left(\boldsymbol{\mu}_{\mathrm{abs}}(\tau) \cdot \mathbf{p}_{\mathrm{abs}}\right)^{2} \mathrm{~d} s(\tau) .
$$

The integral in Eq. (21) depends only on the angle between $\mathbf{p}_{\mathrm{abs}}$ and $\boldsymbol{\mu}_{\mathrm{em}}$, so for this computation we may take, without loss of generality, $\boldsymbol{\mu}_{\mathrm{em}}=\mathbf{k}$ and $\mathbf{p}_{\text {abs }}=(\cos \rho, 0, \sin \rho)$. The circle of absorption dipoles is thus readily parameterized as

$$
\boldsymbol{\mu}_{\mathrm{abs}}(\tau)=(\sin \beta \cos \tau, \sin \beta \sin \tau, \cos \beta)
$$

and so $\left(\boldsymbol{\mu}_{\mathrm{abs}}(\tau) \cdot \mathbf{p}_{\mathrm{abs}}\right)^{2}=(\cos \rho \sin \beta \cos \tau+\sin \rho$ $\cos \beta)^{2}$. Then

$$
\begin{aligned}
\int_{\mathbb{S}}\left(\boldsymbol{\mu}_{\mathrm{abs}}(\tau) \cdot \mathbf{p}_{\mathrm{abs}}\right)^{2} \mathrm{~d} s(\tau)= & \int_{0}^{2 \pi}(\cos \rho \sin \beta \cos \tau \\
& +\sin \rho \cos \beta)^{2} \mathrm{~d} \tau \\
= & \pi\left(\cos ^{2} \rho \sin ^{2} \beta+2 \sin ^{2} \rho \cos ^{2} \beta\right) \\
= & \pi\left[\left(1-\left(\mu_{\mathrm{em}} \cdot \mathbf{p}_{\mathrm{abs}}\right)^{2}\right) \sin ^{2} \beta\right. \\
& \left.+2\left(\boldsymbol{\mu}_{\mathrm{em}} \cdot \mathbf{p}_{\mathrm{abs}}\right)^{2} \cos ^{2} \beta\right]
\end{aligned}
$$

and Eq. (21) becomes

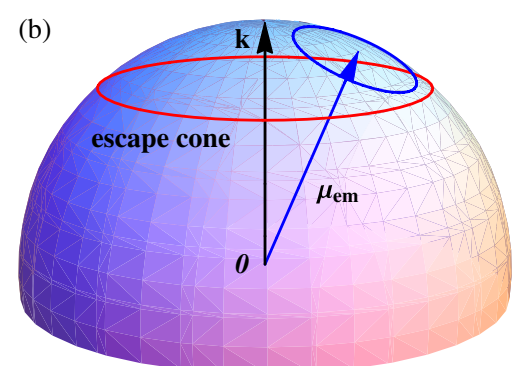

Fig. 6. (Color online) Description of the computational geometry when $\theta_{\mathrm{em}}<\theta_{\mathrm{esc}}$. (a) Case $\theta_{\text {esc }}-\theta_{\mathrm{em}}<\sigma<\theta_{\mathrm{em}}+\theta_{\text {esc }}$. (b) Case $\theta_{e m}<\theta_{\mathrm{esc}}, 0<\sigma<\theta_{\mathrm{esc}}-\theta_{\text {em }}$. 


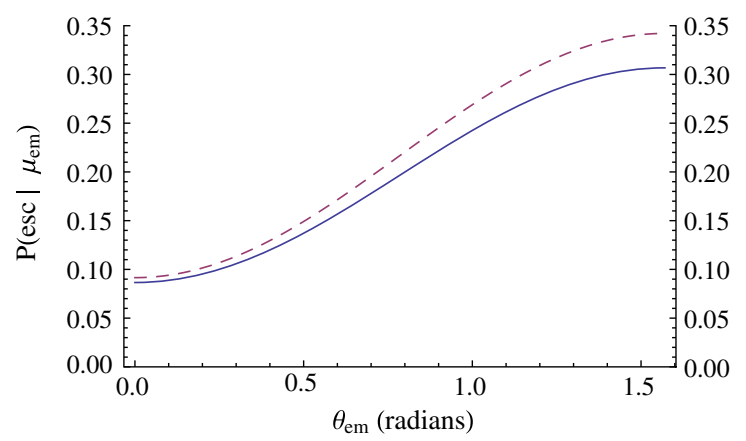

Fig. 7. (Color online) Probability of escape conditional on emission from $\boldsymbol{\mu}_{\mathrm{em}}$. Solid curve: including Fresnel relations; dashed curve: neglecting Fresnel relations.

$$
\begin{aligned}
P\left(\boldsymbol{\mu}_{\mathrm{em}} \mid \mathbf{p}_{\mathrm{abs}}, \mathbf{v}_{\mathrm{abs}}\right)= & \frac{3 q}{8 \pi}\left[\left(1-\left(\boldsymbol{\mu}_{\mathrm{em}} \cdot \mathbf{p}_{\mathrm{abs}}\right)^{2}\right) \sin ^{2} \beta\right. \\
& \left.+2\left(\boldsymbol{\mu}_{\mathrm{em}} \cdot \mathbf{p}_{\mathrm{abs}}\right)^{2} \cos ^{2} \beta\right] .
\end{aligned}
$$

In terms of our chosen parameterizations,

$$
\begin{aligned}
\boldsymbol{\mu}_{\mathrm{em}} \cdot \mathbf{p}_{\mathrm{abs}}= & \cos \alpha_{p}\left(\cos \theta_{\mathrm{ph}} \cos \varphi_{\mu_{e}} \sin \theta_{\mu_{e}}\right. \\
& \left.-\sin \theta_{\mathrm{ph}} \cos \theta_{\mu_{e}}\right)+\sin \alpha_{p} \sin \varphi_{\mu_{e}} \sin \theta_{\mu_{e}} .
\end{aligned}
$$

As mentioned above, the usual approach for the computation of escape-cone losses takes the ratio of the solid angle of the escape cone to the surface area of the sphere, but this is correct only for unpolarized light, averaged over all directions of propagation, and not taking the Fresnel relations into account. To see that the above derivations are consistent with this number, we compute the probability of escape using Eq. (17), averaged over polarization angle $\alpha$ and direction $\mathbf{v}_{\mathrm{em}}$ :

$$
\begin{aligned}
& \frac{1}{4 \pi} \frac{1}{2 \pi} \int_{0}^{2 \pi} \int_{0}^{\pi} \int_{0}^{2 \pi} P\left(\operatorname{esc} \mid \mathbf{v}_{\mathrm{abs}}\left(\theta_{\mathrm{ph}}, \varphi_{\mathrm{ph}}\right), \mathbf{p}_{\mathrm{abs}}\left(\theta_{\mathrm{ph}}, \varphi_{\mathrm{ph}}, \alpha\right)\right) \\
& \quad \times \mathrm{d} \alpha \sin \theta_{\mathrm{ph}} \mathrm{d} \theta_{\mathrm{ph}} \mathrm{d} \varphi_{\mathrm{ph}}=0.2587,
\end{aligned}
$$

in agreement with Eq. (1). (One can make the analogous calculation with the Fresnel relations taken into account, and we find the proportion reduces to 0.2334 .)

\section{Experimental Treatment}

To test the model, experiments were performed measuring escape-cone losses from a $10 \mathrm{~mm} \times$ $10 \mathrm{~mm} \times 27 \mathrm{~mm}$ rectangular PMMA block $\left(n_{1}=1.49\right)$ doped with a low concentration $(\approx 1 \mathrm{ppm})$ of the fluorescent red dye Lumogen $F$ Red 570 (BASF, maximum absorption wavelength $=570 \mathrm{~nm}$, maximum emissionwavelength $=617 \mathrm{~nm}$ in 2-propanol, fluorescent quantum yield $=1.0 \quad[24])$. The angle $\beta=15^{\circ}$ between dye absorption and emission transition moments was separately determined from measurements of the anisotropy, performed according to standard methods with a right-angle fluorometer [22]. Using the setup in Fig. 8, a polarized, collimated

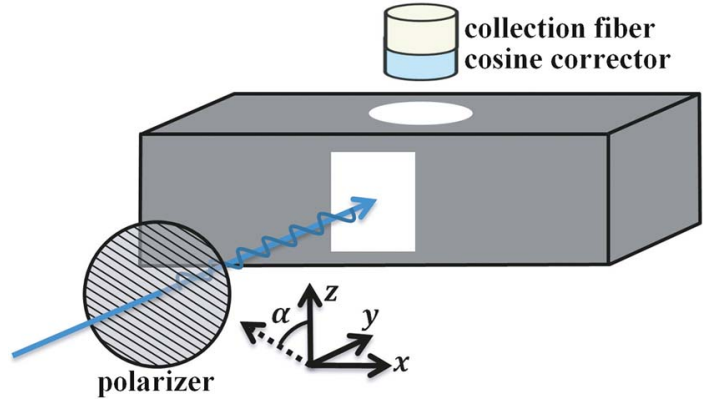

Fig. 8. (Color online) Experimental setup: collimated monochromatic light is polarized at angle $\alpha$ and illuminates a rectangular window on the fluorescent PMMA block. Emitted light escaping out the circular window is collected by a fiber optic terminated by a cosine corrector.

beam of $492 \mathrm{~nm}$ monochromatic light was introduced through one face of the sample and absorbed by dye molecules, and the resulting fluorescent emission was collected by a fiber optic positioned on an orthogonal face. The direction of illumination is parallel to the $y$ axis; this corresponds to $\mathbf{v}_{\mathrm{abs}}=\pi / 2$ in Eq. (5). The experiment was repeated four times, illuminating and collecting from all four large faces, with the results used to compute the $95 \%$ confidence interval in the analysis below. To allow for the capture of rays escaping the sample from the widest possible range of angles, the collection fiber was terminated by a $3.2 \mathrm{~mm}$ diameter cosine corrector located $1.2 \mathrm{~mm}$ from the emission face. The cosine corrector uses a scattering optic to increase the effective numerical aperture of the fiber so that all rays intersecting it, regardless of their incident direction, are detected with essentially equal probability. Collected light was passed through a monochromator to a photomultiplier detector; the resulting fluorescence spectra were then integrated over the dye's emission range to give a signal proportional to the number of photons escaping the sample.

All faces of the block were blackened with the exception of two openings that were centered in adjacent facets: one $4.4 \mathrm{~mm} \times 4.9 \mathrm{~mm}$ rectangular opening for illumination and a second $5.5 \mathrm{~mm}$ diameter circular opening for emission. The background-corrected extinction of the sample at the peak absorbance wavelength over a $1 \mathrm{~cm}$ path length was 0.016 , meaning only $\sim 4 \%$ of the incident light was absorbed by the dye throughout the full thickness of the sample. The extinction at the peak emission wavelength was 20 -fold smaller still; consequently to a very good approximation, we neglect repeated absorption/emission and treat every absorbed photon as interacting with a single luminophore.

The polarization of incident light was fixed by a rotatable polarizer, and emission spectra were recorded in $10^{\circ}$ increments from $\alpha=0^{\circ}$ to $\alpha=180^{\circ}$, where $0^{\circ}$ corresponds to the electric field plane oriented parallel to the $z$ axis in Fig. 8. A least-squares fit of the resulting data to a curve of the form $-a \cos (2(\alpha-b))+c$ yields $a=-8536.2, b=-3.7^{\circ}$, 
and $c=39206$. The nonzero value for $b$ indicates that the calibration of $\alpha=0$ was slightly off, and so we shift the experimental data accordingly. The resulting least-squares fit is then, of course, $I(\alpha)=$ $-8536.2 \cos (2 \alpha)+39206$.

If we denote the linearization $\hat{I}(x)=-8356.2 x+$ 39206, $(x=\cos (2 \alpha))$, then analysis of the residuals for the linearized problem shows them to be distributed normally, and the $R^{2}$ statistic for the fit is 0.92 . Given the normality of the residuals, we compute 95\% confidence intervals for the coefficients $a$ and $c$ to be

$$
a \in[7995,9187] \text { and } c \in[38746,39610] .
$$

At confidence 95\%, we calculate the resulting envelope for the linear regression line to be

$$
-8591 x+39178 \pm 3755 \sqrt{0.013+0.025(x-0.052)^{2}} .
$$

The linearized data and regression line, together with the envelope of $95 \%$ confidence, are shown in Fig. 9.

\section{Comparison of Experimental Data to Theoretical Calculations}

Because the experimental data has not been normalized with respect to any given reference intensity, any multiple of $I(\alpha)$ equally well represents the dependence of escape-cone intensity on angle $\alpha$. A characteristic quantity is thus the ratio $I(0) / I(90)=$ 0.642 of the minimum to the maximum intensities. When we compute [Eq. (5)] the corresponding theoretical ratio, we find

$$
\frac{P\left(\operatorname{esc} \mid \alpha=0^{\circ}\right)}{P\left(\operatorname{esc} \mid \alpha=90^{\circ}\right)}=0.692 .
$$

(We include the Fresnel relations because the face of the sample opposite the integrating sphere is blackened.) This number, however, cannot be used in comparison with the experimental result. The reason for this is the theoretical computation in Eq. (5) assumes that all light that escapes the sample is measured by the fiber optic. This is clearly not the

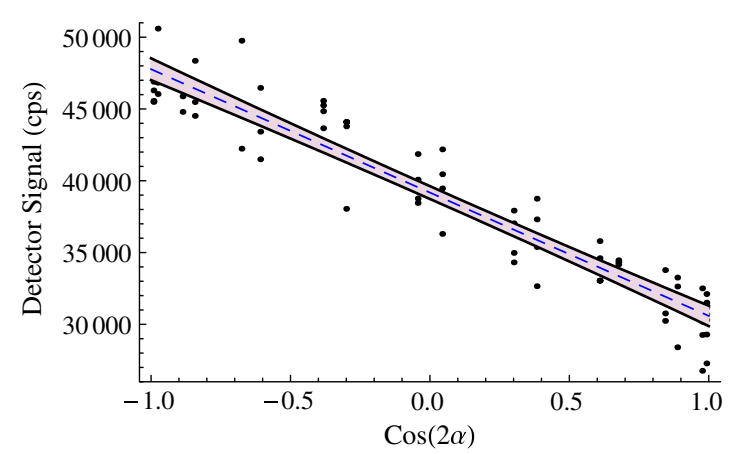

Fig. 9. (Color online) Linearized experimental data. The straight dashed blue line is the linear regression line to this data, and the shaded region is the $95 \%$ confidence interval. case in the experimental setup. For example, emission from close to the bottom $(z=-0.50 \mathrm{~cm})$ of the sample which reaches the collection window is constrained to a cone with angle $\arctan (0.275 / 1.0)=$ $15.4^{\circ}$ due to the finite size of the circular emission opening, while the full escape cone has an angle of $42^{\circ}$. Similarly, approximately half of the emission from close to the edge of the collection window will not be in the direction of the aperture. To obtain a correct theoretical comparison we must integrate, over the full illuminated volume, the probability that an emitted photon would escape [according to Eq. (5)] and be in such a direction that, after refracting at the face $z=0.50 \mathrm{~cm}$ and propagating to $z=0.62 \mathrm{~cm}$, the photon intersects the cosine corrector.

Implementing the described integration, we obtain

$$
\begin{gathered}
P(\operatorname{esc} \mid \alpha)=-0.0104 \cos (2 \alpha)+0.051, \\
\text { for which } \frac{P\left(\operatorname{esc} \mid \alpha=0^{\circ}\right)}{P\left(\operatorname{esc} \mid \alpha=90^{\circ}\right)}=0.658 .
\end{gathered}
$$

To understand this with respect to the confidence intervals derived above, we must scale $P(\operatorname{esc} \mid \alpha)$ judiciously. We calculate the scalar $\gamma$ so that $\gamma(-0.0104 x+0.051)$ minimizes the sum of the squares of the residuals; that is

$$
\begin{aligned}
\gamma & =\operatorname{argmin} \sum_{i=1}^{N}\left(\gamma\left(-0.0104 x_{i}+0.051\right)-\hat{I}_{i}\right)^{2} \\
& =775864,
\end{aligned}
$$

where $N=76$ is the total number of measurements and $\hat{I}_{i}$ is the unscaled data. The resulting scaled theoretical model is then $-8081 \cos (2 \alpha)+39207$. This is shown in Fig. 10 (the solid purple curve) together with the nonlinear least-squares fit $I(\alpha)$ to the experimental data (the blue dashed curve) and the translation of the $95 \%$ confidence region of Fig. $\underline{9}$ to this setting (the shaded region). We thus find that the theoretical model is in good agreement with the observed experimental results.

\section{Derivation of Introductory Example}

We assume here that the absorption and emission dipoles coincide. The emission dipoles are assumed to be distributed according to

$$
\begin{aligned}
\boldsymbol{\mu}_{\mathrm{abs}} & =\boldsymbol{\mu}_{\mathrm{em}} \sim h_{\mu}(\theta) \sin \theta \mathrm{d} \theta \mathrm{d} \varphi \\
& =\frac{e^{c_{2} P_{2}(\cos \theta)} \sin \theta}{2 \pi \int_{0}^{\pi} e^{c_{2} P_{2}\left(\cos \theta^{\prime}\right)} \sin \theta^{\prime} \mathrm{d} \theta^{\prime}} \mathrm{d} \theta \mathrm{d} \varphi,
\end{aligned}
$$

where $P_{2}$ is the second Legendre polynomial. We first compute the probability that a dipole $\boldsymbol{\mu}(\theta, \varphi)$ absorbs (and therefore emits) a photon, conditional on the fact that the incoming light has direction $\mathbf{v}_{0}=$ $(0,0,-1)$ and has polarization perpendicular to this; using the parameterization of Eq. (3) for the polarization $\mathbf{p}(\pi, \alpha)$, 


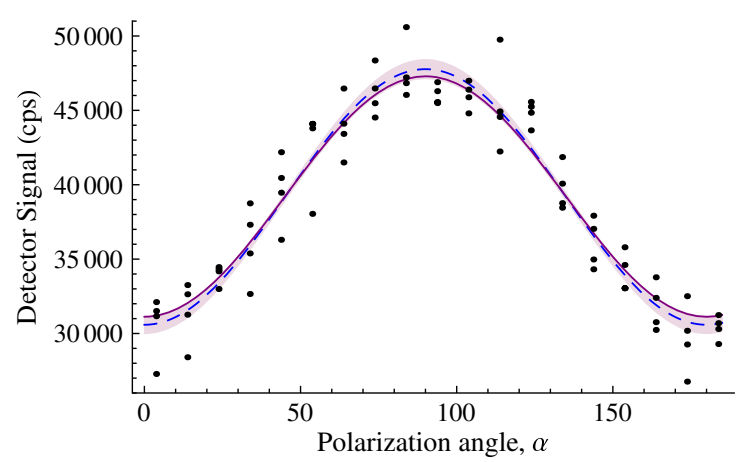

Fig. 10. (Color online) Experimental data points, least squares fit $I(\alpha)$ (dashed blue curve), scaled theoretical model (solid purple curve), and $95 \%$ confidence region (shaded).

$$
\begin{aligned}
& P\left(\boldsymbol{\mu}(\theta, \varphi) \mid \mathbf{v}_{0}, \mathbf{p}(\pi, \alpha)\right) \\
& \quad=\frac{(\boldsymbol{\mu}(\theta, \varphi) \cdot \mathbf{p}(\pi, \alpha))^{2} h_{\mu}(\theta) \sin \theta \mathrm{d} \theta \mathrm{d} \varphi}{\int_{0}^{2 \pi} \int_{0}^{\pi}\left(\boldsymbol{\mu}\left(\theta^{\prime}, \varphi^{\prime}\right) \cdot \mathbf{p}(\pi, \alpha)\right)^{2} h_{\mu}\left(\theta^{\prime}\right) \sin \theta^{\prime} \mathrm{d} \theta^{\prime} \mathrm{d} \varphi^{\prime}},
\end{aligned}
$$

and then

$P\left(\boldsymbol{\mu}(\theta, \varphi) \mid \mathbf{v}_{0}\right.$, random $\left.\mathbf{p}\right)=\frac{1}{2 \pi} \int_{0}^{2 \pi} P\left(\boldsymbol{\mu}(\theta, \varphi) \mid \mathbf{v}_{0}, \mathbf{p}(\pi, \alpha)\right) \mathrm{d} \alpha$.

A photon emitted from a dipole $\boldsymbol{\mu}$ is emitted in a direction that makes an angle $\hat{\theta}$ with $\boldsymbol{\mu}$ according to the distribution $3 /(8 \pi) \sin ^{2} \hat{\theta} \sin \hat{\theta} \mathrm{d} \hat{\theta} \mathrm{d} \hat{\varphi}$ [where $(\hat{\theta}, \hat{\varphi})$ are spherical coordinates with $\hat{\theta}=0$ corresponding to $\boldsymbol{\mu}$ ]. Thus, the probability of being emitted with direction $\mathbf{v}$, given that it was emitted from a dipole distributed according to Eq. (32), is

$$
P(\mathbf{v} \mid \boldsymbol{\mu})=\frac{3}{8 \pi}\left(1-(\boldsymbol{\mu} \cdot \mathbf{v})^{2}\right),
$$

and the probability that it has emission direction $\mathbf{v}$ given that it was emitted at the first absorption/ emission event is

$$
\begin{aligned}
P(\mathbf{v} \mid 1) & :=P(\mathbf{v} \mid \text { first emission event }) \\
& =\int_{S^{2}} P(\mathbf{v} \mid \boldsymbol{\mu}) P\left(\boldsymbol{\mu} \mid \mathbf{v}_{0}, \text { random } \mathbf{p}\right) \mathrm{d} A(\boldsymbol{\mu}) .
\end{aligned}
$$

Finally, to be in the escape cone,

$$
\begin{aligned}
& P \text { (esc|first emission event) } \\
& \quad=2 \int_{0}^{2 \pi} \int_{0}^{\theta_{\mathrm{esc}}} P(\mathbf{v}(\theta, \varphi) \mid 1) \sin \theta \mathrm{d} \theta \mathrm{d} \varphi .
\end{aligned}
$$

Further absorption/emission events are handled similarly. We describe the computation inductively. Let $P\left(\boldsymbol{\mu}\left(\theta_{\mu}, \varphi_{\mu}\right) \mid n\right)$ be the probability that $\boldsymbol{\mu}$ is the absorbing and emitting dipole at the $n$th absorption/ emission event $\left[P\left(\mu\left(\theta_{\mu}, \varphi_{\mu}\right) \mid 1\right)\right.$ is given by Eq. (32)]. We first compute the probability density function for the direction $\mathbf{v}(\theta)$ and polarization $\mathbf{p}(\theta, \alpha)$ conditional to the fact that it has been emitted at the $n$th event. This is given by

$$
P(\mathbf{v}, \mathbf{p} \mid n)=\frac{f(\theta, \alpha) \mathrm{d} \alpha \mathrm{d} \theta \mathrm{d} \varphi}{\int_{0}^{2 \pi} \int_{\theta_{\text {esc }}-\theta_{\text {esc }}}^{\pi} \int_{0}^{2 \pi} f\left(\theta^{\prime}, \alpha^{\prime}\right) \mathrm{d} \alpha^{\prime} \mathrm{d} \theta^{\prime} \mathrm{d} \varphi^{\prime}},
$$

where

$$
f(\theta, \alpha)=\int_{\text {possible } \mu} P(\mathbf{v}, \mathbf{p} \mid \boldsymbol{\mu}) P(\boldsymbol{\mu} \mid n) \mathrm{d} s(\boldsymbol{\mu}) ;
$$

here the integral is over the circle of possible $\mu$ that can give rise to the velocity-polarization pair. This can be parameterized as

$$
\boldsymbol{\mu}_{\mathrm{poss}}(\tau ; \theta, \alpha)=\cos (\tau) \mathbf{v}(\theta)+\sin (\tau) \mathbf{p}(\theta, \alpha) .
$$

The first term in the above integral (37) is

$$
P(\mathbf{v}(\theta), \mathbf{p}(\theta, \alpha) \mid \boldsymbol{\mu}(\tau))=\frac{1}{2 \pi^{2}}\left(1-(\mathbf{v}(\theta) \cdot \boldsymbol{\mu}(\tau ; \theta, \alpha))^{2}\right) .
$$

The second term in Eq. (37) is assumed to be known from the previous step. Having determined the distribution of the polarizations of the photons emitted at the $n$th event, we can compute the distribution of the $(n+1)$ st absorbing and emitting dipoles, $P(\mu \mid n+1)$. This is found by

$$
\begin{aligned}
P(\boldsymbol{\mu} \mid n+1)= & \int_{0}^{2 \pi} \int_{\theta_{\mathrm{esc}}}^{\pi-\theta_{\mathrm{esc}}} \int_{0}^{2 \pi} P(\boldsymbol{\mu} \mid \mathbf{v}(\theta), \mathbf{p}(\theta, \alpha)) \\
& \times P(\mathbf{v}, \mathbf{p} \mid n) \sin \theta \mathrm{d} \alpha \mathrm{d} \theta \mathrm{d} \varphi,
\end{aligned}
$$

where

$$
\begin{aligned}
& P\left(\boldsymbol{\mu}\left(\theta_{\mu}, \varphi_{\mu}\right) \mid \mathbf{v}(\theta), \mathbf{p}(\theta, \alpha)\right) \\
& \quad=\frac{\left(\boldsymbol{\mu}\left(\theta_{\mu}, \varphi_{\mu}\right) \cdot \mathbf{p}(\theta, \alpha)\right)^{2} h_{\mu}\left(\theta_{\mu}\right) \sin \left(\theta_{\mu}\right) \mathrm{d} \theta_{\mu} \mathrm{d} \varphi_{\mu}}{\int_{0}^{2 \pi} \int_{0}^{\pi}\left(\boldsymbol{\mu}\left(\theta^{\prime}, \varphi^{\prime}\right) \cdot \mathbf{p}(\theta, \alpha)\right)^{2} h_{\mu}\left(\theta^{\prime}\right) \sin \left(\theta^{\prime}\right) \mathrm{d} \theta^{\prime} \mathrm{d} \varphi^{\prime}} .
\end{aligned}
$$

Finally, as in Eqs. (34) and (35),

$$
\begin{aligned}
& P(\mathbf{v} \mid n+1):=P(\mathbf{v} \mid(n+1) \text { st emission event }) \\
&=\int_{S^{2}} P(\mathbf{v} \mid \boldsymbol{\mu}) P(\boldsymbol{\mu} \mid n+1) \mathrm{d} A(\boldsymbol{\mu}), \\
& P(\mathbf{v} \mid \boldsymbol{\mu})=\frac{3}{8 \pi}\left(1-(\boldsymbol{\mu} \cdot \mathbf{v})^{2}\right), \\
& P(\operatorname{esc} \mid(n+1) \text { st emission event }) \\
&=2 \int_{0}^{2 \pi} \int_{0}^{\theta_{\text {esc }}} P(\mathbf{v}(\theta, \varphi) \mid n+1) \sin \theta \mathrm{d} \theta \mathrm{d} \varphi .
\end{aligned}
$$

This work was supported by the National Science Foundation under Grant No. DMR-1035512. The authors wish to thank Brad Johnson for useful discussions. 


\section{References}

1. G. J. Keil, "Radiance amplification by a fluorescence radiation converter," Appl. Phys. 40, 3544-3547 (1969).

2. W. H. Weber and J. Lambe, "Luminescent greenhouse collector for solar radiation," Appl. Opt. 15, 2299-2300 (1976).

3. J. S. Batchelder, A. H. Zewail, and T. Cole, "Luminescent solar concentrators. 1: theory of operation and techniques for performance evaluation," Appl. Opt. 18, 3090-3110 (1979).

4. J. S. Batchelder, A. H. Zewail, and T. Cole, "Luminescent solar concentrators. 2: experimental and theoretical analysis of their possible efficiencies," Appl. Opt. 20, 3733-3754 (1981).

5. M. I. Barnik, V. G. Vasil'chenko, S. V. Golovkin, A. M Medvedkov, A. S. Solov'ev, and S. G. Yudin, "Scintillation properties of materials based on liquid crystals in static and dynamic states," Instrum. Exp. Tech. 43, 602-611 (2000).

6. G. Baur, A. Stieb, and G. Meier, "Polarized fluorescence of dyes oriented in room temperature nematic liquid crystals," Mol. Cryst. Liq. Cryst. 22, 261-269 (1973).

7. Z. Chen and T. M. Swager, "Synthesis and characterization of fluorescent acenequinones as dyes for guest-host liquid crystal displays," Org. Lett. 9, 997-1000 (2007).

8. X. Zhang, R. Yamaguchi, K. Moriyama, M. Kadowaki, T Kobayashi, T. Ishi-i, T. Thiemann, and S. Mataka, "Highly dichroic benzo-2,1,3-thiadiazole dyes containing five linearly $\pi$-conjugated aromatic residues, with fluorescent emission ranging from green to red, in a liquid crystal guest-host system," J. Mater. Chem. 16, 736-740 (2006).

9. C. J. Koester and E. Snitzer, "Amplification in a fiber laser," Appl. Opt. 3, 1182-1186 (1964).

10. P. P. C. Verbunt and M. G. Debije, "Progress in luminescent solar concentrator research: solar energy for the built environment," in World Renewable Energy Congress (Linköping University Electronic Press, 2011), p. 2751-2758.

11. W. G. J. H. M. van Sark, K. W. J. Barnham, L. H. Slooff, A. J. Chatten, A. Büchtemann, A. Meyer, S. J. McCormack, R. Koole, D. J. Farrell, R. Bose, E. E. Bende, A. R. Burgers, T. Budel, J. Quilitz, M. Kennedy, T. Meyer, C. De Mello Donegá, A. Meijerink, and D. Vanmaekelbergh, "Luminescent solar concentrators-a review of recent results," Opt. Express 16, 21773-21792 (2008)
12. V. V. Popov and V. N. Yakimenko, "State of the art of prospects for investigations of luminescent solar concentrators," J. Appl. Spectrosc. 62, 573-577 (1995).

13. M. G. Debije and P. P. C. Verbunt, "Thirty years of luminescent solar concentrator research: solar energy for the built environment," Adv. Energy Mater. 2, 12-35 (2012).

14. W. A. Shurcliff and R. C. Jones, "The trapping of fluorescent light produced within objects of high geometrical symmetry," J. Opt. Soc. Am. 39, 912-916 (1949).

15. P. P. C. Verbunt, C. W. M. Bastiaansen, D. J. Broer, and M. G. Debije, "The effect of dyes aligned by liquid crystals on luminescent solar concentrator performance," presented at the 24th European Photovoltaic Solar Energy Conference, Hamburg, 21-25 September 2009.

16. C. L. Mulder, P. D. Reusswig, A. M. Velázquez, H. Kim, C. Rotschild, and M. A. Baldo, "Dye alignment in luminescent solar concentrators: I. Vertical alignment for improved waveguide coupling," Opt. Express 18, A79-A90 (2010).

17. C. L. Mulder, P. D. Reusswig, A. P. Beyler, H. Kim, C. Rotschild, and M. A. Baldo, "Dye alignment in luminescent solar concentrators: II. Horizontal alignment for energy harvesting in linear polarizers," Opt. Express 18, A91-A99 (2010).

18. M. G. Debije, "Solar energy collectors with tunable transmission," Adv. Funct. Mater. 20, 1498-1502 (2010).

19. R. W. MacQueen, Y. Y. Cheng, R. G. C. R. Clady, and T. W. Schmidt, "Towards an aligned luminophore solar concentrator," Opt. Express 18, A161-A166 (2010).

20. S. McDowall, B. L. Johnson, and D. L. Patrick, "Simulations of luminescent solar concentrators: effects of polarization and fluorophore alignment," J. Appl. Phys. 108, 053508 (2010).

21. H. Coles and S. Morris, "Liquid-crystal lasers," Nat. Photonics 4, 676-685 (2010).

22. J. R. Lakowicz, Principles of Fluorescence Spectroscopy, 3rd ed. (Springer, 2006).

23. R. H. Lehmberg, "Radiation from an N-atom system. I. General formalism," Phys. Rev. A 2, 883-888 (1970).

24. L. R. Wilson and B. S. Richards, "Measurement method for photoluminescent quantum yields of fluorescent organic dyes in polymethyl methacrylate for luminescent solar concentrators," Appl. Opt. 48, 212-220 (2009). 